\title{
Human Mast Cells and Mastocytosis: Harnessing MicroRNA Expression as a New Approach to Therapy?
}

\author{
Lorenzo Deho' • Silvia Monticelli
}

Received: 9 October 2009/ Accepted: 6 January 2010/Published online: 29 May 2010

(c) L. Hirszfeld Institute of Immunology and Experimental Therapy, Wroclaw, Poland 2010

\begin{abstract}
MicroRNAs (miRNAs) are short, non-coding RNAs that have emerged as key post-transcriptional regulators in a wide variety of organisms and critical cellular processes. Because any one miRNA can regulate the expression of a distinct set of genes, differential miRNA expression can shape the repertoire of proteins that are actually expressed during development, differentiation, or disease. To understand what goes wrong when a cell becomes transformed requires knowledge of the processes that ensure normal development. It is now clear that miRNAs may act as oncogenes and/or tumor suppressors within gene regulatory networks, thereby contributing to the development of cancer. Mast cells are longlived cells, widely distributed throughout vascularized tissues, in particular near surfaces that are exposed to the environment (such as skin, airways, and the gastrointestinal tract), where they contribute to bacterial clearance, enhancement of adaptive immune responses, modulation of inflammation, and the degradation of toxic peptides and venoms. Here we review current knowledge in the field of mast-cell differentiation and disease in humans and mice and discuss future directions and links between mast-cell differentiation, oncogenic transformation, and microRNAs as well as possible new points of entry for therapeutic intervention.
\end{abstract}

Keywords Mast cells - MicroRNA - Mastocytosis · Gene regulation · Disease

\section{Abbreviations \\ MC Mast cells \\ miRNA MicroRNA}

L. Deho' · S. Monticelli ( $\bowtie)$

Institute for Research in Biomedicine,

Via Vincenzo Vela 6, 6500 Bellinzona, Switzerland

e-mail: silvia.monticelli@irb.unisi.ch

\section{Introduction}

MicroRNAs (miRNAs) are a class of small, non-coding RNAs found in organisms ranging from nematodes to humans and even viruses. They function mostly as endogenous translational repressors of protein-coding genes by binding to target sites in the $3^{\prime}$ untranslated region ( $3^{\prime}$ UTR) of messenger RNAs. miRNAs are derived from short hairpins of 60-70 nt (pre-miRNAs) that are cleaved in the nucleus from longer primary transcripts (pri-miRNAs) by the Microprocessor complex, which includes the RNAse III endonuclease Drosha. Pre-miRNAs are exported to the cytoplasm, where the mature $\sim 22-n t$ miRNA is excised by Dicer and loaded onto the RNA-induced silencing complex (RISC). The miRNA-charged RISC binds to the $3^{\prime}$ UTR of target mRNAs and catalyzes mRNA translational inhibition, destabilization, or degradation (reviewed in Kanellopoulou and Monticelli 2008; Kim et al. 2009).

Cells in the mammalian hematopoietic system express a multitude of miRNA species which may or may not be shared between different cell types or development stages, but only a few of which are specific to the hematopoietic system (Monticelli et al. 2005; Neilson et al. 2007; Wu et al. 2007). The generation of the immune system from hematopoietic stem cells involves ordered events of lineage commitment, differentiation, proliferation, and cell migration. Within such processes, miRNAs appear ideally suited to adjust protein concentrations rapidly in cells, allowing complex cellular transitions to subsequent developmental and activation stages. Accordingly, certain miRNAs are expressed in a stage-specific fashion (Monticelli et al. 2005; Neilson et al. 2007; Wu et al. 2007) and miRNA control has recently emerged as a critical regulatory principle in the mammalian immune system. Indeed, it was 
shown that genetic ablation of the miRNA machinery (Chong et al. 2008; Muljo et al. 2005) as well as loss or deregulation of certain individual miRNAs severely compromised immune development and led to immune disorders (reviewed in Kanellopoulou and Monticelli 2008; Xiao and Rajewsky 2009). With the recent identification of hundreds of genes that produce non-coding RNA transcripts with no significant open reading frame, it has become evident that the genomic complexity of any cell is far greater than expected (reviewed in Pipkin and Monticelli 2008). Consistent with the discovery that miRNAs modulate gene expression, altered miRNA expression has been found to affect cancer development. By negatively regulating proto-oncogenes, miRNAs could act as tumor suppressors; conversely, by inhibiting tumor suppressors, miRNAs could function as oncogenes. Indeed, both increased and decreased miRNA abundance has been observed in cancer cells compared with normal tissue.

Mast cells are cells of the innate immune system that reside in most tissues of the body and derive from hematopoietic precursors in the bone marrow. Mast cells function as effector and immunoregulatory cells in IgEassociated allergic disorders as well as in certain innate and adaptive immune responses (Kalesnikoff and Galli 2008). Abnormal, dysregulated, and clonal accumulation of mast cells gives rise to disorders such as mastocytosis. These disorders can have a clinical course variably ranging from asymptomatic for years to highly aggressive and rapidly devastating. Within this scenario, since it is now clear that miRNAs can act as oncogenes, and also contribute to the normal regulation of most systems and pathways so far analyzed, it is tempting to hypothesize that miRNAs may also contribute to the uncontrolled growth of mast cells or their progenitors. Here we review current knowledge in the field, focusing, when possible, on human mast cells, although due to the paucity of available data, our conclusions by necessity will depend in part on results of studies using non-human mast cells.

\section{Distribution, Differentiation, and Activation of Mast Cells}

Mast cells are long-lived cells derived from hematopoietic progenitor cells. They usually do not circulate in mature form in the blood, as their differentiation and maturation take place in the tissues where they will ultimately reside; nevertheless, upon stimulation, mast cells can reenter the cell cycle and proliferate. Specifically, an increase in number or tissue distribution as well as changes in phenotypical characteristics of mast cells can occur during $\mathrm{T}$ helper 2 (Th2) lymphocyte responses (Galli et al. 2008a). In mammals and other vertebrates, mast cells are widely distributed throughout vascularized tissues, particularly beneath epithelia and in close proximity to blood vessels, nerves, smooth muscle cells, mucus-producing glands, and hair follicles (Galli et al. 2005b), and are especially prominent in areas exposed to the environment (skin, mucosal airways, and the gastrointestinal tract), where pathogens and allergens are frequently encountered. Mast cells are critical effector cells in allergic disorders; however, recent studies have shown that these cells are heterogeneous and can produce an array of pro- and antiinflammatory mediators, act as antigen-presenting cells, and express a spectrum of co-stimulatory molecules, indicating that mast cells are far more functionally diverse than previously imagined and can function as immunoregulatory cells (Benoist and Mathis 2002; Galli et al. 2005a, b).

Mast cells can participate in a wide variety of physiological and pathological processes as a result of their activation through a variety of receptors. However, two of the best-studied receptors expressed on differentiated mast cells are the high-affinity $\mathrm{IgE}$ receptor $(\mathrm{Fc} \varepsilon \mathrm{RI})$ and c-Kit (Fig. 1). Both of these receptors have important roles in the differentiation, survival, proliferation, and function of mast cells (reviewed in Kalesnikoff and Galli 2008). In particular, mast cells are well known as key effector cells in IgEassociated immune responses. In appropriately sensitized hosts, encounter with a specific antigen will induce mast cells to undergo activation dependent on specific $\operatorname{IgE}$ bound to the FceRI. The cytoplasm of mature mast cells is characteristically full of granules containing preformed mediators such as proteoglycans, proteases, and histamine. Upon cell activation, these preformed mediators are released into the extracellular milieu. The chemical properties of the granules can be used to stain the cells with toluidine blue or other similar stains that will turn characteristically purple in the presence of the content of the granules (Fig. 1).

The types and properties of the endopeptidases stored in the granules vary with the organism and the final tissue where the mast cell will ultimately reside and terminate its differentiation. Based on the expression of these enzymes, human mast cells have been broadly classified into two major classes: those expressing tryptase only (this type of cells is called also $\mathrm{MC}_{\mathrm{T}}$ ), which tend to be located in the mucosa of organ systems, and those expressing both chymase and tryptase $\left(\mathrm{MC}_{\mathrm{TC}}\right)$, which tend to be abundant in the dermis (Fig. 1).

Human mast cells can be differentiated in vitro from precursors derived from cord blood or bone marrow as well as from hematopoietic progenitors separated from peripheral blood (Holm et al. 2008). While the main growth factor for rodent mast cells is IL-3, human mast cells seem to be relatively more IL-3 independent and require more strictly SCF (the ligand for the c-Kit receptor) for their 


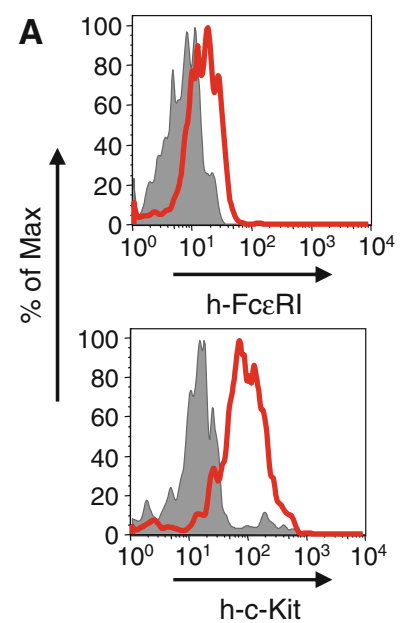

Fig. 1 Human mast cells differentiated in vitro express specific mastcell markers. a Committed hCD $133^{+}$progenitors were separated from peripheral blood and differentiated in vitro for 5 weeks. In the first 3 weeks the cells were cultured in Stem Span serum-free medium supplemented with hIL-3, hIL-6, and hSCF, while in the last 2 weeks the cytokine cocktail was changed to hIL-6, hSCF, and hIL-4. The cells were than stained for the surface mast-cell markers FceRI and c-KIT (red lines; in grey the negative controls for the staining). b The same cells as in a were also stained with toluidine blue to visualize the cytoplasmic granules of differentiated mast cells (in purple, as

growth and survival (Galli et al. 2008b; Saito et al. 2006). An example of the resulting phenotype of human mast cells differentiated in vitro in our lab is shown in Fig. 1 (Deho' and Monticelli, unpublished). Interestingly, these in vitro differentiated cells contained tryptase in their cytoplasmic granules, but not chymase, indicating an $\mathrm{MC}_{\mathrm{T}}$-like phenotype that is considered still rather immature (Galli et al. 2008a). Cultured human mast cells with an $\mathrm{MC}_{\mathrm{TC}}$ phenotype (i.e. expressing a high amount of chymase as well as tryptase) can be obtained starting from cord blood precursors treated with IL-4 in the latest stages of the differentiation process (Toru et al. 1998a), indicating a possible pathway of development from tryptase single-positive into tryptase/chymase double-positive cells and suggesting that this maturation process is promoted by IL-4.

Murine mast cells can be easily differentiated not only from bone marrow, but also from embryonic stem cells (Garrington et al. 2000; Monticelli et al. 2004; Tsai et al. 2000); these embryonic stem-derived mast cells represent a very important tool to study the effect of genetic alteration in mast-cell differentiation and function. Recently, murine as well as human differentiated adult cells have been reprogrammed directly to pluripotency by ectopic expression of four transcription factors (Oct4, Sox2, Klf4, and Myc) to yield induced pluripotent stem (iPS) cells (Park et al. 2008; Takahashi and Yamanaka 2006). Patient-specific iPS cells hold great therapeutic potential: indeed, it has been recently shown that on correction of the genetic defect, somatic cells from Fanconi anemia patients can be opposed to the blue of the nuclei and the pale blue of the cytoplasm of other cell types). c Top The same cells as in a were stained for the enzymes tryptase (red) and chymase (green), that are normally present in cytoplasmic granules, while the nuclei were counterstained with DAPI (blue). The in vitro differentiated cells turned out to be tryptase $^{+}$and chymase ${ }^{-}$. Bottom As a control for the stainings, a tissue sample from human ileum mucosa was used. Mast cells that are tryptase $^{+}$are red, while those that are both tryptase ${ }^{+}$and chymase ${ }^{+}$ are yellow. The numbers at the top left corner indicate the magnification used

reprogrammed to pluripotency to generate patient-specific iPS cells (Raya et al. 2009). Most importantly, Raya and colleagues showed that genetically corrected Fanconi anemia-specific iPS cells can give rise to hematopoietic progenitors of the myeloid and erythroid lineages that are phenotypically normal, i.e. disease free. In order to study the effect of genetic alteration in human mast-cell differentiation and function, a possibility would be to obtain iPS cells from patients with altered mast-cell functions (which in principle could range from allergic inflammation to aggressive mastocytosis) and study the differentiation and function of these cells in vitro.

\section{Mast Cells in Inflammation}

Mast cells are mostly famous for their association with acute manifestations of hypersensitivity reactions, allergic asthma, allergic rhinitis, and anaphylaxis. Following activation through the IgE receptor FceRI, mast cells not only release mediators that are pre-stored in the cytoplasmic granules, but also initiate de novo synthesis and release of several lipid-derived molecules as well as cytokines, chemokines, and growth factors. These products may be released when mast cells are activated via IgE- or IgG-dependent mechanisms and may also be produced in response to stimulation by bacterial products through Toll-like receptors. A number of potential functions have been associated with various mast-cell proteases, including 
bronchoconstriction, and degradation of fibrinogen and extracellular matrix proteins.

Several studies have shown that mast cells can produce an array of pro- and anti-inflammatory mediators and express a spectrum of co-stimulatory molecules, indicating that mast cells are functionally diverse and can function as immunoregulatory cells (Benoist and Mathis 2002; Galli et al. $2005 a, b)$. Indeed, mast cells can exert positive or negative immunomodulatory effects on other immune cells by influencing their recruitment, survival, development, phenotype, or function and thereby enhance or suppress the initiation, magnitude, and/or duration of immune responses (reviewed in Galli et al. 2008a). Molecules produced by mast cells with possible immunomodulatory functions include members of the tumor necrosis factor (TNF) family as well as cytokines that can influence T-cell functions such as IL-4, IL-5, IL-6, and IL-13. Mast cells can also mediate negative immunomodulatory functions in vivo by producing IL-10 (Grimbaldeston et al. 2007). Moreover, mast cells express MHC class I and II molecules on their surface and they have been reported to be able to process and present antigen in vitro (Galli et al. 2008b). However, the in vivo relevance of some of these molecules remains to be determined. It also needs to be highlighted that many of these studies were performed on murine mast cells and it is now clear that these may not always predict the human mast-cell response. For example, while mouse mast cells produce significant amounts of IL-4, human mast cells may not (Toru et al. 1998b).

Although blood monocytes, tissue macrophages, and Kupffer cells are the best-known sources of TNF, mast cells (at least in the mouse) are the only cell type capable of storing pre-synthesized TNF. Because of this unique capability, mast cells provide the only readily available source of TNF within peripheral tissues during the early course of infection. During infection, peripheral tissue mast cells regulate lymph node hypertrophy through the rapid secretion of TNF (McLachlan et al. 2003). Interestingly, it has recently been shown that peripheral murine mast cells, upon activation, release stable submicrometer heparinbased particles containing TNF and other proteins. These insoluble complexes increase the normally short half-life of TNF, enter lymphatic vessels, and rapidly traffic to the draining lymph nodes. This physiological drug delivery system facilitates communication between peripheral sites of inflammation and remote secondary lymphoid tissues (Kunder et al. 2009).

Excessive or inappropriate mast-cell activation also contributes to the development of certain allergic or autoimmune diseases, which are mediated by allergen- or autoantigen-specific effector T cells (Galli et al. 2005b). No clinically available pharmacological agents can specifically inhibit either mast-cell degranulation or mast-cell mediator release. Understanding these processes in human cells could help in developing mast-cell inhibitors of selective mediator release with novel therapeutic applications (Theoharides et al. 2007). Apart from this "negative" role, it is important to remember that mast cells reside at the crossroads between the external environment, the blood vasculature, and the lymphatic system. They are therefore ideally situated to orchestrate events during the earlier stages of immune responses and it seems likely that this is their physiological role in mammalian biology.

\section{Mast-Cell Diseases}

Although mast cells have been mostly studied for their role in allergic disorders, they have also been implicated in a range of other diseases, including diabetes (Geoffrey et al. 2006; Liu et al. 2009), obesity and angiogenesis (Liu et al. 2009), experimental autoimmune encephalomyelitis (Tanzola et al. 2003), and rheumatoid arthritis (Eklund 2007). Mastocytosis is a mast-cell disorder characterized by dysregulated and clonal accumulation of mast cells and/or mast-cell products in organs such as the skin, gastrointestinal tract, bone marrow, liver, spleen, and lymph nodes, although any tissue may be affected. Mastocytosis can occur at any age and the exact prevalence is unknown. There are many variants of this disease, presenting a wide range of symptoms and prognoses. The two main variants are classified as cutaneous, most common in children, and systemic mastocytosis and they are now believed to be in part dependent on somatic mutation or inherited polymorphisms. In cutaneous mastocytosis there is no evidence of systemic involvement and three major variants are now recognized: (i) urticaria pigmentosa/macuolopapular mastocytosis is the most frequent form and in children the lesions are usually larger and papular. In this variant, mast cells assume a characteristic spindle shape and fill the papillary dermis. In adults the lesions are red and disseminated. (ii) Diffuse cutaneous mastocytosis is less frequent than the previous variant and is present almost exclusively in children. The skin is thick and may have a peau chargine (orange peel) appearance. (iii) Mastocytoma of the skin occurs as a single lesion exclusively in infants and without predilection of site. Vice versa, systemic mastocytosis is characterized by the involvement of at least one extracutaneous organ, even without skin lesions. There are many variants of systemic mastocytosis, including indolent systemic mastocytosis, bone marrow mastocytosis, mast-cell leukemia, and mastcell sarcoma (WHO 2008). Prognosis depends on the disease category and currently no specific cure is available. In children, cutaneous mastocytosis usually has a favorable outcome and may regress spontaneously. In adults, however, cutaneous lesions do not regress and are often associated with systemic mastocytosis, usually the indolent variant. 
Patients with aggressive diseases, including mast-cell leukemia, survive only a few months, whereas patients with indolent systemic mastocytosis have a normal life expectancy (WHO 2008).

Although some genetic alterations in mastocytosis have been described (for example, the $\mathrm{D} 816 \mathrm{~V}$ gain-of-function mutation in the c-KIT proto-oncogene), little is known concerning pathogenetic factors that contribute to the development of disease variants and disease progression. Additional defects, that remain to be identified, are likely to contribute to the uncontrolled growth of mast cells and/ or their progenitors and to the resulting adverse clinical course (Metcalfe 2008; Valent et al. 2005).

Allergic disorders, such as anaphylaxis, hay fever, eczema, and asthma, afflict roughly $25 \%$ of people in the developed world. Asthma is a complex disease of the airways whose incidence, morbidity, and mortality have increased dramatically over the past two decades and continue to do so, particularly in industrialized countries. In atopic asthmatics, the immune system responds to harmless environmental allergens which are typically ignored by non-atopic immune systems. Key to the development of asthma is the priming of allergen-specific $\mathrm{CD} 4^{+} \mathrm{T}$ lymphocytes and their differentiation to a Th2 phenotype. These cells orchestrate atopic asthmatic inflammation through the secretion of cytokines, particularly IL-4, IL-5, and IL-13, of which IL-4 in particular drives IgE production by B cells. Following such sensitization, subsequent exposure to the specific allergen initiates a secondary response characterized by rapid onset of mucosal edema, increase in airway smooth muscle tone, and airway narrowing. This response is induced by IgE-mediated mastcell degranulation and the associated release of inflammatory mediators, including histamine, prostaglandins, and leukotrienes. While early- and late-phase reactions to an allergen can resolve fully without treatment, chronic allergic inflammation is a persistent inflammation that occurs at sites of repeated allergen exposure and can lead to altered functions of the affected organs (Tillie-Leblond et al. 2005). It is therefore important to understand the characteristics and consequences of acute and chronic allergic inflammation, and in particular to explore how mast cells can contribute to several features of this immunological reactivity (Galli et al. 2008b). Moreover, while a role for miRNAs has been clearly established for the development and functions of other cell types and for diseases such as cancer, their role in mast-cell physiology as well as in asthma remains to be elucidated.

\section{Mast Cells and miRNAs}

The molecular network that regulates mast-cell differentiation and function has not been investigated as widely as it has been in other hematopoietic cells. There are still many mechanisms of regulation that need to be fully elucidated to understand how external stimuli can lead to an appropriate physiological response by mast cells. Such information could be used to determine potential therapeutic targets for the control of mast-cell activation in inflammatory diseases, allergy, and asthma. Our group has already shown that some selected miRNAs might be important for murine mast-cell differentiation (Monticelli et al. 2005) as well as the proliferation of differentiated mast cells (Mayoral et al. 2009). Specifically, we identified a family of two miRNAs, miR-221-222, which is upregulated following activation of differentiated murine bone marrow-derived mast cells (BMMCs) (Mayoral et al. 2009). This is one of the first examples of miRNAs whose expression changes as a result of acute cell activation, as opposed to cell differentiation, and implies a role for miRNAs not only during cell differentiation and to maintain cell identity, but also in cellular effector functions and/or proliferation. Indeed, using both bioinformatics and experimental approaches, we characterized some transcriptional requirements (in particular, we suggested involvement of the transcription factors NF- $\kappa \mathrm{B}, \mathrm{NFAT}$, and PLZF) for the miR-221-222 gene, and through analysis of the pattern of DNaseI hypersensitivity we identified potential cis-regulatory regions that might control the expression of miR-221-222 in mast cells. Furthermore, by overexpressing miR-221-222 individually or in combination, we found that these two miRNAs cooperate in regulating cell cycle and cell proliferation in mast cells. Interestingly, overexpression of miR-221-222 had a modest effect on the expression of the known target $\mathrm{p} 27^{\mathrm{Kip} 1}$; we showed that such partial effect on $\mathrm{p} 27^{\mathrm{Kip} 1}$ was due to a splice variant of $\mathrm{p} 27^{\mathrm{Kip} 1}$ that does not contain miR-221-222 binding sites in its 3' UTR. Finally, cell-cycle regulation is likely to be a more general effect of miR-221-222, as transgenic expression of miR-221-222 from BAC clones in murine embryonic stem cells dramatically reduced cell proliferation and severely impaired their accumulation. Our study provided further insights into miR-221222 transcriptional regulation as well as evidence that miR-221-222 regulates cell-cycle checkpoints in mast cells in response to acute activation stimuli. Following up on those studies, by lentivirally transducing primary BMMCs we could show that miR-221-222 are not only important in mast-cell proliferation, but have pleiotropic effects on essential mast-cell processes such as migration, survival, and homing (Mayoral and Monticelli, unpublished). Nevertheless, a direct link between mast cells, miRNAs, and mast-cell diseases remains to be uncovered, especially concerning the human system.

\section{miRNAs in Therapy?}

The importance of miRNAs in fine-tuning gene expression is highlighted by the finding that changes in the abundance of a 
single miRNA can affect the levels of expression of hundreds of proteins (Baek et al. 2008; Selbach et al. 2008). It is therefore not surprising that a single dysregulated miRNA can have profound effects on the state of the cell. There are indeed now numerous examples linking dysregulated expression of miRNAs to cancer (Croce 2009; Kanellopoulou and Monticelli 2008) and miRNAs are increasingly viewed as potential therapeutic targets (see also Fig. 2). While restoring miRNA expression can be used in cases of miRNAs that function as onco-suppressors, it is important to be able to dampen miRNA expression or block their function in cases in which their overexpression favors cellular oncogenic transformation (i.e. "onco-miRs"). To achieve this, new technologies are emerging (Fig. 2). Such technologies provide a versatile tool to investigate miRNA functions utilizing artificial systems to either upregulate or ablate miRNA expression or to affect the expression of a target mRNA by means of the RNA interference (RNAi) pathway. Double-stranded RNA oligonucleotides (miRNA "mimics") or antisense oligonucleotides to the miRNA sequence (2'-O-methyl oligonucleotides, or locked nucleic acids) can be effectively used to upregulate or block the expression of miRNAs in a wide variety of cells and systems. The drawback with using oligos is that the system is intrinsically transient. Other systems to overexpress or block long-term miRNA function use vectors based on lentiviruses, adenoviruses, and retroviruses. Vectors to overexpress miRNAs usually contain the miRNA sequence plus $\sim 200 \mathrm{bp}$ of genomic sequence on each side to ensure proper processing. Instead, vectors to inhibit miRNA functions are called decoys, sponges, or erasers and usually contain multiple miRNA target sites cloned downstream from a reporter gene expressed from a strong promoter (Fig. 2), often of viral origin (Haraguchi et al. 2009). The overexpression of artificial miRNA target sites in the cells prevents the miRNA of choice from regulating its natural targets (Brown and Naldini 2009; Brown et al. 2006). Finally, RNAi is the process of mRNA degradation that is induced by double-stranded RNA in a sequence-specific manner. The specificity and effectiveness of RNAi makes it a valuable research tool: synthetic dsRNA introduced into cells can down-modulate the expression of specific genes of interest (Siomi and Siomi, 2009), while miRNA-based vectors can be used to express short hairpin RNAs to reduce the levels of expression of selected oncogenes through the RNAi pathway (Chang et al. 2006). Recently, Kota et al. (2009) demonstrated in a mouse model of liver cancer that ectopic expression of a single miRNA, miR-26a, can reverse disease progression. In this particular study, the delivery of an miR-26a-expressing construct in vivo was achieved by using an adeno-associated virus (AAV) vector. AAV vectors are particularly suitable as gene therapy vectors as they can be produced in large quantities and do not integrate into the
A Modulating miRNA expression:

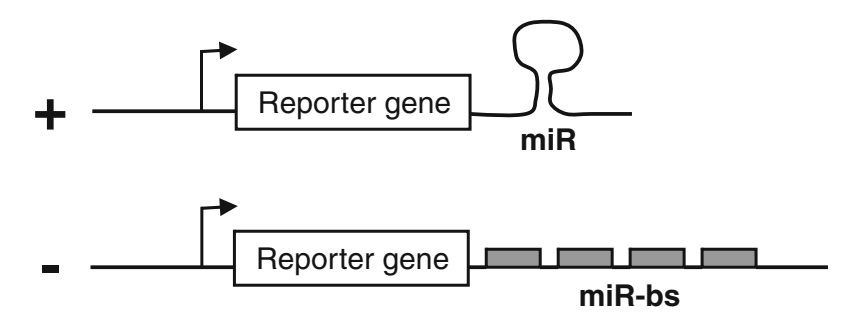

B Silencing mRNA: RNAi

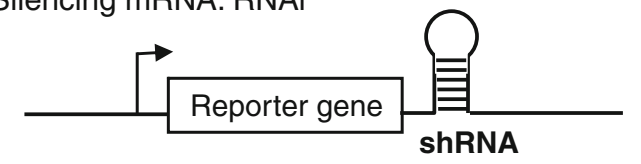

Fig. 2 Vectors used to modulate miRNA or mRNA expression. a Schematic representation of vectors commonly used to modulate miRNA levels to enhance $(+)$ or silence $(-)$ miRNA expression. $(+)$ Vector a vector to overexpress miRNAs requires a promoter (usually Pol II dependent) (arrow) and the miRNA sequence with $\sim 200$ bp of genomic sequence on each side to ensure proper processing. In this and in all the other vectors shown, a reporter gene can be used to identify and/or select transduced cells. (-) Vector a vector to functionally ablate the miRNA of choice is made by cloning several miRNA binding sites downstream from a reporter gene. Upon transcription, this gives rise to synthetic miRNA targets, therefore preventing the miRNA of choice from regulating its natural targets. b Vectors similar to the ones described in a can be used also to express short hairpin RNA (shRNA) for silencing a specific oncogenic mRNA through the RNAi pathway

host genome. This means that they will eventually be eliminated, thereby minimizing the potential of vectormediated toxicities. This report represents an important step toward the potential application of miRNA-based therapy for the treatment of cancers. In particular, correction of a genetic defect in mast-cell diseases by altering miRNA levels or by using miRNA-based technology holds promise for the correction of such diseases.

\section{Conclusions}

Mastocytosis may occur at any age, familial occurrence is unusual, and the exact prevalence of mastocytosis and its variants is unknown. Moreover, treatment of mastocytosis is based on amelioration of symptoms with pharmacological agents that inhibit the actions of mediators identified as mast cell derived, especially histamine and leukotrienes, but so far no standard therapy for patients with aggressive forms of disease has been defined (Valent et al. 2005; WHO 2008). It is therefore essential to uncover some of the molecular networks that may be at the basis of mastocytosis development and to provide further tools for diagnosis and prognosis. Ultimately, we look forward to understanding how perturbation of miRNA-related networks 
may apply to disease conditions, with the hope that they may lend diagnostic insight or lead to novel entry points for therapeutic intervention.

Acknowledgments This work was supported by a Swiss National Science Foundation grant (no. 3100A0_121991) to S. Monticelli. We would also like to thank the European School of Oncology Foundation (Lugano, Switzerland) for support.

\section{References}

Baek D, Villen J, Shin C et al (2008) The impact of microRNAs on protein output. Nature 455:64-71

Benoist C, Mathis D (2002) Mast cells in autoimmune disease. Nature 420:875-878

Brown BD, Naldini L (2009) Exploiting and antagonizing microRNA regulation for therapeutic and experimental applications. Nat Rev Genet 10:578-585

Brown BD, Venneri MA, Zingale A et al (2006) Endogenous microRNA regulation suppresses transgene expression in hematopoietic lineages and enables stable gene transfer. Nat Med 12:585-591

Chang K, Elledge SJ, Hannon GJ (2006) Lessons from nature: microRNA-based shRNA libraries. Nat Methods 3:707-714

Chong MM, Rasmussen JP, Rudensky AY et al (2008) The RNAseIII enzyme Drosha is critical in $\mathrm{T}$ cells for preventing lethal inflammatory disease. J Exp Med 205:2005-2017

Croce CM (2009) Causes and consequences of microRNA dysregulation in cancer. Nat Rev Genet 10:704-714

Eklund KK (2007) Mast cells in the pathogenesis of rheumatic diseases and as potential targets for anti-rheumatic therapy. Immunol Rev 217:38-52

Galli SJ, Kalesnikoff J, Grimbaldeston MA et al (2005a) Mast cells as "tunable" effector and immunoregulatory cells: recent advances. Annu Rev Immunol 23:749-786

Galli SJ, Nakae S, Tsai M (2005b) Mast cells in the development of adaptive immune responses. Nat Immunol 6:135-142

Galli SJ, Grimbaldeston M, Tsai M (2008a) Immunomodulatory mast cells: negative, as well as positive, regulators of immunity. Nat Rev Immunol 8:478-486

Galli SJ, Tsai M, Piliponsky AM (2008b) The development of allergic inflammation. Nature 454:445-454

Garrington TP, Ishizuka T, Papst PJ et al (2000) MEKK2 gene disruption causes loss of cytokine production in response to $\operatorname{IgE}$ and c-kit ligand stimulation of ES cell-derived mast cells. EMBO J 19:387-5396

Geoffrey R, Jia S, Kwitek AE et al (2006) Evidence of a functional role for mast cells in the development of type 1 diabetes mellitus in the BioBreeding rat. J Immunol 177:7275-7286

Grimbaldeston MA, Nakae S, Kalesnikoff J et al (2007) Mast cell-derived interleukin 10 limits skin pathology in contact dermatitis and chronic irradiation with ultraviolet B. Nat Immunol 8:1095-1104

Haraguchi T, Ozaki Y, Iba H (2009) Vectors expressing efficient RNA decoys achieve the long-term suppression of specific microRNA activity in mammalian cells. Nucleic Acids Res 37:43

Holm M, Andersen HB, Hetland TE et al (2008) Seven week culture of functional human mast cells from buffy coat preparations. J Immunol Methods 336:213-221

Kalesnikoff J, Galli SJ (2008) New developments in mast cell biology. Nat Immunol 9:1215-1223

Kanellopoulou C, Monticelli S (2008) A role for microRNAs in the development of the immune system and in the pathogenesis of cancer. Semin Cancer Biol 18:79-88
Kim VN, Han J, Siomi MC (2009) Biogenesis of small RNAs in animals. Nat Rev Mol Cell Biol 10:126-139

Kota J, Chivukula RR, O'Donnell KA et al (2009) Therapeutic microRNA delivery suppresses tumorigenesis in a murine liver cancer model. Cell 137:1005-1017

Kunder CA, St John AL, Li G et al (2009) Mast cell-derived particles deliver peripheral signals to remote lymph nodes. J Exp Med 206:2455-2467

Liu J, Divoux A, Sun J et al (2009) Genetic deficiency and pharmacological stabilization of mast cells reduce diet-induced obesity and diabetes in mice. Nat Med 15:940-945

Mayoral RJ, Pipkin ME, Pachkov M et al (2009) MicroRNA-221-222 regulate the cell cycle in mast cells. J Immunol 182:433-445

McLachlan JB, Hart JP, Pizzo SV et al (2003) Mast cell-derived tumor necrosis factor induces hypertrophy of draining lymph nodes during infection. Nat Immunol 4:1199-1205

Metcalfe DD (2008) Mast cells and mastocytosis. Blood 112:946-956

Monticelli S, Solymar DC, Rao A (2004) Role of NFAT proteins in IL13 gene transcription in mast cells. J Biol Chem 279:36210 36218

Monticelli S, Ansel KM, Xiao C et al (2005) MicroRNA profiling of the murine hematopoietic system. Genome Biol 6:R71

Muljo SA, Ansel KM, Kanellopoulou C et al (2005) Aberrant T cell differentiation in the absence of Dicer. J Exp Med 202:261-269

Neilson JR, Zheng GX, Burge CB et al (2007) Dynamic regulation of miRNA expression in ordered stages of cellular development. Genes Dev 21:578-589

Park IH, Zhao R, West JA et al (2008) Reprogramming of human somatic cells to pluripotency with defined factors. Nature 451:141-146

Pipkin ME, Monticelli S (2008) Genomics and the immune system. Immunology 124:23-32

Raya A, Rodriguez-Piza I, Guenechea G et al (2009) Diseasecorrected haematopoietic progenitors from Fanconi anaemia induced pluripotent stem cells. Nature 460:53-59

Saito H, Kato A, Matsumoto K et al (2006) Culture of human mast cells from peripheral blood progenitors. Nat Protoc 1:2178-2183

Selbach M, Schwanhausser B, Thierfelder N et al (2008) Widespread changes in protein synthesis induced by microRNAs. Nature 455:58-63

Siomi H, Siomi MC (2009) On the road to reading the RNAinterference code. Nature 457:396-404

Takahashi K, Yamanaka S (2006) Induction of pluripotent stem cells from mouse embryonic and adult fibroblast cultures by defined factors. Cell 126:663-676

Tanzola MB, Robbie-Ryan M, Gutekunst CA et al (2003) Mast cells exert effects outside the central nervous system to influence experimental allergic encephalomyelitis disease course. J Immunol 171:4385-4391

Theoharides TC, Kempuraj D, Tagen M et al (2007) Differential release of mast cell mediators and the pathogenesis of inflammation. Immunol Rev 217:65-78

Tillie-Leblond I, Gosset P, Tonnel AB (2005) Inflammatory events in severe acute asthma. Allergy 60:23-29

Toru H, Eguchi M, Matsumoto R et al (1998a) Interleukin-4 promotes the development of tryptase and chymase double-positive human mast cells accompanied by cell maturation. Blood 91:187-195

Toru H, Pawankar R, Ra C et al (1998b) Human mast cells produce IL-13 by high-affinity IgE receptor cross-linking: enhanced IL-13 production by IL-4-primed human mast cells. J Allergy Clin Immunol 102:491-502

Tsai M, Wedemeyer J, Ganiatsas S et al (2000) In vivo immunological function of mast cells derived from embryonic stem cells: an approach for the rapid analysis of even embryonic lethal mutations in adult mice in vivo. Proc Natl Acad Sci USA 97:9186-9190 
Valent P, Akin C, Sperr WR et al (2005) Mastocytosis: pathology, genetics, and current options for therapy. Leuk Lymphoma $46: 35-48$

WHO (2008) WHO classification of tumours of haematopoietic and lymphoid tissues. International Agency for Research on Cancer, Lyon
Wu H, Neilson JR, Kumar P et al (2007) miRNA profiling of naive, effector and memory CD8 T cells. PLoS One 2:e1020

Xiao C, Rajewsky K (2009) MicroRNA control in the immune system: basic principles. Cell 136:26-36 